\title{
Erratum to: LncRNA-N1LR Enhances Neuroprotection Against Ischemic Stroke Probably by Inhibiting p53 Phosphorylation
}

\author{
Zhuomin $\mathrm{Wu}^{1} \cdot$ Ping $\mathrm{Wu}^{2} \cdot$ Xialin $\mathrm{Zuo}^{3} \cdot \mathrm{Na} \mathrm{Yu}^{4} \cdot$ Yixin Qin ${ }^{1} \cdot \mathrm{Qian}_{\mathrm{Xu}^{1}}{ }^{1} \mathrm{Shuai}_{\mathrm{He}^{1}}{ }^{1}$ \\ Bohong Cen ${ }^{1} \cdot$ Wenjie Liao ${ }^{1} \cdot \operatorname{Aimin} \mathrm{Ji}^{1}$
}

Published online: 4 January 2017

(C) Springer Science+Business Media New York 2016

Erratum to: Mol Neurobiol.

DOI: 10.1007/s12035-016-0246-Z

The original paper of this article unfortunately contains mistakes.

In Fig. 4c, the northern blot analysis validated the size of the IncRNA-N1LR. When we performed the northern blot analysis, all the RNA bands from different samples appeared to be in the same size, which were consistent with the result of the RACE. However, in these results, there was a band that was brighter than others, and we guessed the sample from this brighter band was polluted by the sample overexpressing N1LR. Unfortunately, this brighter band mistakenly appeared in Fig. 4c. So we would like to present the correct Fig. 4c. In addition, the TTC stained image of "siRNA-NC" in Fig. 6c was mistakenly replaced by another image from the sample of the mouse suffering $1.5 \mathrm{~h}$ of MCAO and $24 \mathrm{~h}$ of reperfusion. With this, the correct Fig. $6 \mathrm{c}$ is hereby presented.

The online version of the original article can be found at http://dx.doi. org/10.1007/s12035-016-0246-z

Aimin Ji

aiminji_007@163.com

1 Center for Drug Research and Development, Zhujiang Hospital, Southern Medical University, Industrial avenue253, Guangzhou 510282, China

2 Pharmacy Department, Chengdu First People's Hospital/Chengdu Integrated TCM \& Western Medicine Hospital, Chengdu, China

3 Institute of Neurosciences and the Second Affiliated Hospital of Guangzhou Medical University, Guangzhou, China

4 The Second Clinical College of Southern Medical University, Guangzhou, China 
Fig. 4 Information about lncRNA-N1LR. a The genomic location of IncRNA-N1LR. LncRNA-N1LR is located on chromosome 9 in mice and shares an overlapping sequence with the 5' UTR of Nck1. siRNA was designed to target the region of lncRNA-N1LR outside of the overlapping sequence with $5^{\prime}$ UTR of Nck1. b The full-length sequence of IncRNA-N1LR obtained by RACE analysis. Agarose gel electrophoresis and the sequencing of the PCR product from the 5'-RACE and 3'RACE procedure revealed a putative transcriptional start and end site of lncRNA-N1LR. The nucleotide sequence of the fulllength lncRNA-N1LR is given below. c Northern blot analysis showed the length of the lncRNAN1LR fragment in the cortex of mice subjected to I/R-induced injury a $100,540 \mathrm{k}$ $100,530 k$ $100,520 \mathrm{k}$ $100,510 k$ $100,500 \mathrm{k}$

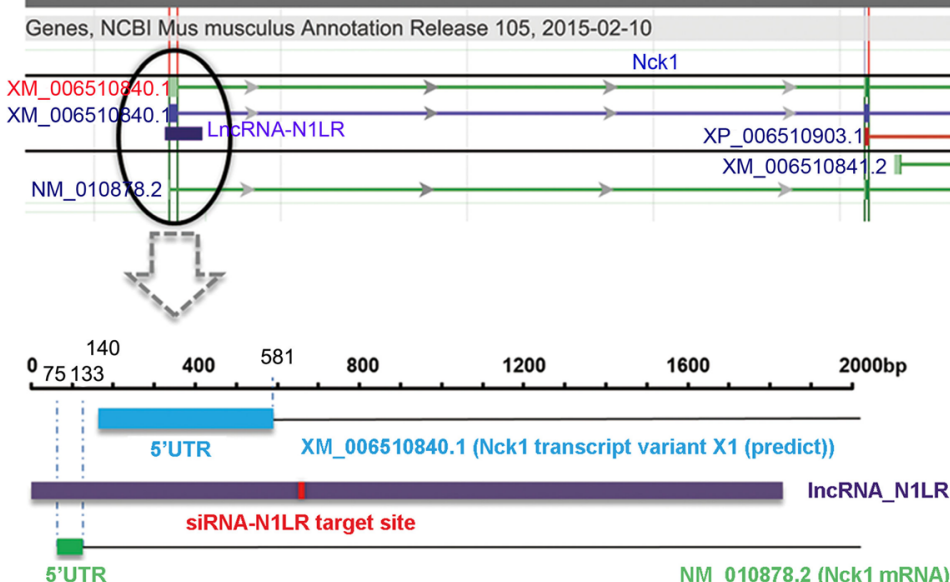

b
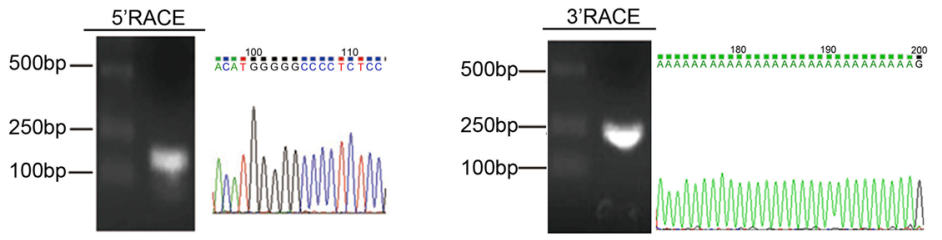

1 ACATGggGgC CCCTCTCCGA GAGCTACGCG gCGgCGCAGC ACAGgCCTCG TGCGgTAACG 61 GCATCCGGGC AGCCGCGGTG GCGTCCCGGT GCGGTCCTCA GGTGACTGGA GCCGAGGGCT 121 CGGCGGAGGG AAGGTGAGAC GGCCTCGCTC GCGTGCGGAC ACACGCACAG TCCTTTCGGT 181 CTCCCGGTCG GTCCGCTCTG CCTGCTAGTC TCTAGTCGCT TCCTCTTTCC CCGGCGAAGT 241 TGGCCCGGGG GCGCCGTCTG CTGGCGGCGG AGCGGAGCGG AGGGGGCGGG GAGGCCGCTC 301 GGCGGAGCTC GGGTGTCCGC GGTGCAGCCC GGTGACCCCG CGGCCTCTGG CCTGAGCTGC 361 GAGCGCCGGT CGGGGAGGCG GGCAGGCAAG TGTGCGGCGC GCTGCCTCGG CGGTTCTCCG 421 CAGAAAGCCC TGTCGGCGGT CTGTGTCGGT GTCCGAGGCC GGGACGGTAG GCTGGCAGGA 481 AGGGCGCTCT CACCTCCAGC CGCCAGCCTG AGGAAGCCGA GAGGGGGGAC ATCCTCGGGG 541 CGCGCCCTCT CCTCCCCCTC CCGTTCCGGA GCAGGAGAGA TGTGGGTGTC CGGGGGCTGT 601 TGAGGTGGGG TTGGTCCAGG AAAAGCAGTC CTCTGGGTTG GTTTCGTACT GTTGTCGTAC 661 GGGTTTCCGT GCTAGGTCGG TTCAGTGTTA ACAGCTGGGT TGGAAGGAAG CTGGTTTTTT 721 GTTCAGGGTT GAGGACCGTA ACATGACCGG TTAACGTCGA GCCGAGCTGA AGTCACCCGA 781 AGAAAAATAC TGCCCTTAGT TAGGAAAGAA TTCACAGGAG TTAGGAAAGA ACGAGATGAA 841 GAAAACTTCC TGACCCCATC TTGGGTGGGG AGCAGTGAGA ATCCAGGGTT CGGCCAAAAA 901 AACGGGTCAA GTTTTGCAAG AAGCGTTCCA TTTTCAAAAG CTTTGGTAGC TTGATGATGA 961 TCAATCAGCC TTAACGGTTT CTTAAGCACC TGGAAATCAA TTGTGTGCTT TCAGCCTTTA 1021 GCGACGTCAC AACATGAACA GAGAAATAGT CCAAGGAGAA AGTTTTGATT GATCATTTTC 1081 AGTCTTGTTT TTAAATAGAT ATTCTCTTCA CATCGTGATA GTTAAGTCAA AGCGTATGCG 1141 TTGGTTGATT AGTACTTTTT TTTTCATCGT GGCCACATGT GGTGCTTTCT CTGGGAAAAC 1201 TTCTGTAGGA ATTGGCTAGA GTAACTAAAG CGTACATTGT GTTGATGTCT CCGCTGTATG 1261 TATGAGCATT ATGTTTGAAA AGATTTGCCT CCCCAGCAAG CATTTTAAAT TGTTTAGGTT 1321 TCCAAGCCCA ACAGATCCGT GTATTAATAA GTCGACACGT TAATGTATTG CCTTATTAAA 1381 CATTGGGTGA TAGTTTATGC TGCTGATTTT CTAATTTGAG AACTTCGTTC TAGAGTTCAA 1441 GGCTTGTTGT GATTATTATT GTAAATAAAT TCCTTCTCAG GAGAACTTGA AAAAAAATGA 1501 ATTAACTCTG GCAATGTGGA AGAATGGATG CTTTCAATGC CAGAAAATCA AATGATTATT 1561 TCACTGTTAA GTAAAATTGG AGGAAACGTG AACTGAGCAT TTAACAAATC CAACAGTTTG 1621 ACGACAATTT GACAGGCCTG CCTGATTCTC ACTTTAACCT ATCAAGGAAA TAGAACTTCA 1681 AAGTCTTCCC TTCACAACTG ATGCCCCACG CCTCCAAGCT GTCTTTCATT AGCGATACAT 1741 TAGTAGCATG CTTTCAGGAA CCCAGACATA AACCTTAACA TTGTACTGTT TTTTTGTAGT 1801 TGTTTTCTTT AAAAAAAAAA AAAAAAAAAA AAAAAAAAA
C 23130bparker N1LR 9416bp $6557 \mathrm{bp}$ $4361 \mathrm{bp}$ $2322 \mathrm{bp}$ 
Fig. 6 LncRNA-N1LR

ameliorates ischemic brain injury in vivo. a Changes in IncRNA-

N1LR expression in the corresponding location of the ischemic penumbra in mice. $\mathbf{b}$ Quantitative analysis of neurobehavioral scores in mice. The error bar represents the maximum (upper) and the minimum (lower) score. c, $\mathbf{d}$ Representative images showing TTC-stained brain sections (c) and the quantitative analysis of brain infarct volume in mice following $2 \mathrm{~h}$ of MCAO and $24 \mathrm{~h}$ of reperfusion (d). e A diagram of the brain sections. An infarct area is shown as a stippled area. The black box represented the region used for histological analysis. f, g Apoptosis in the ischemic penumbra was analyzed by TUNEL staining. Representative images showed TUNEL-positive cells in tissues from the different groups (f) and quantitative analysis indicated that siRNA-N1LR enhanced apoptosis and that Ad-N1LR significantly decreased apoptosis in mouse cortical neurons (g). h, i

Representative images showing Nissl staining to determine neural cell loss in the ischemic penumbra (h) and the quantitative analysis of the effect of IncRNA-N1LR on neural cell loss (i). The data represent the mean \pm SEM. $* P<0.05$ and $* * P<0.01$ versus Sham; ${ }^{\#} P<0.05$ and ${ }^{\# \#} P<0.01$ versus normal saline; ${ }^{\mathrm{a}} P<0.05$ and ${ }^{\text {aa }} P<0.01$ versus siRNA-NC or Ad-NC. $N=5$ per group a

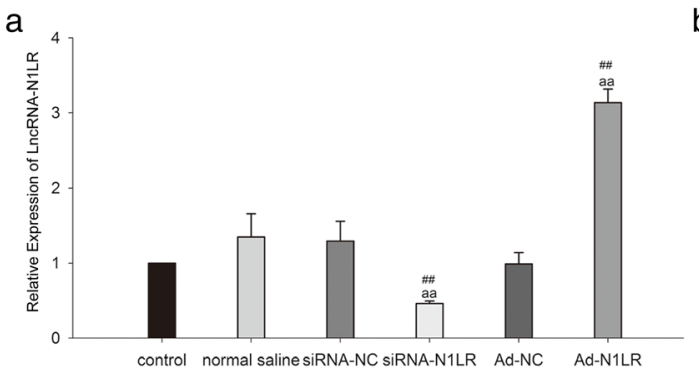

C

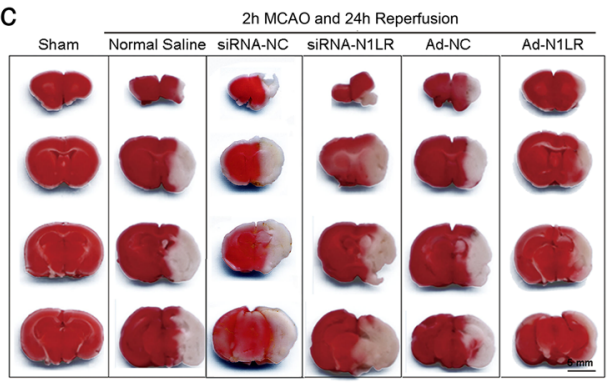

e

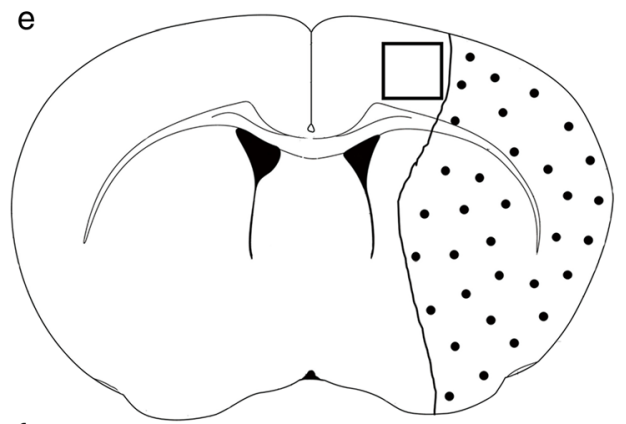

f sham

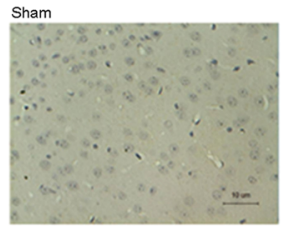

Normal Saline
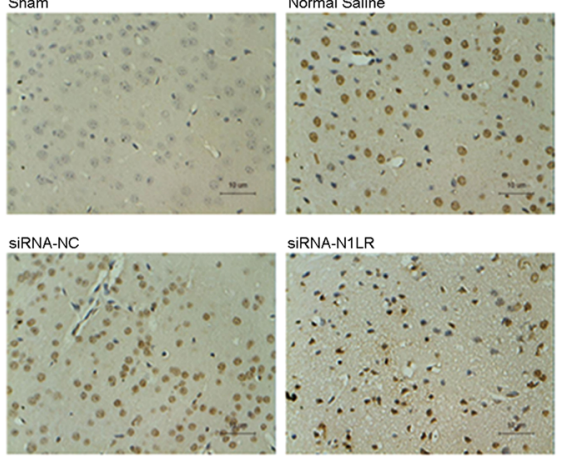

SIRNA-N1LR
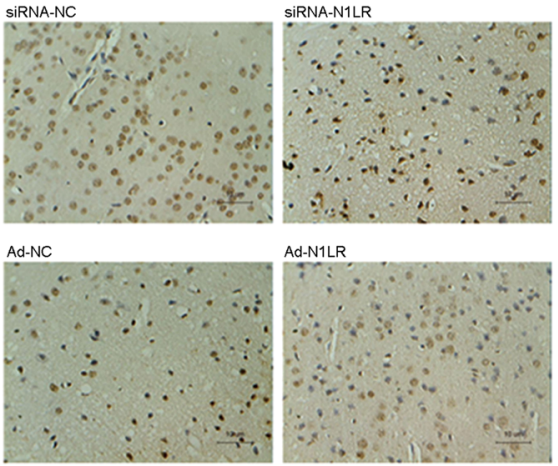

Ad-N1LR

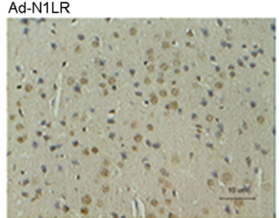

g

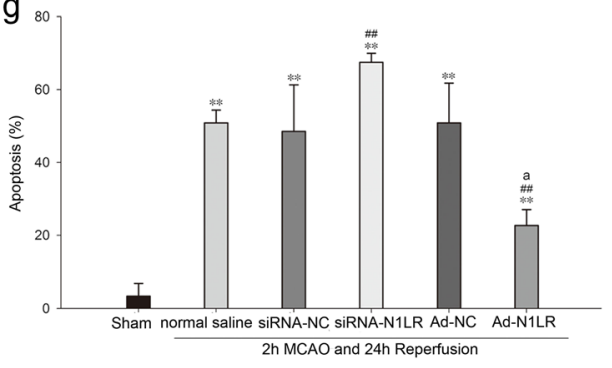

b

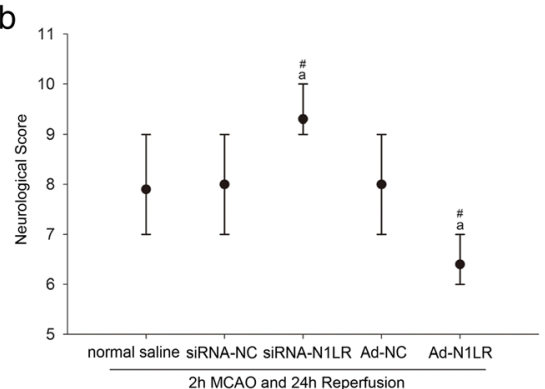

d

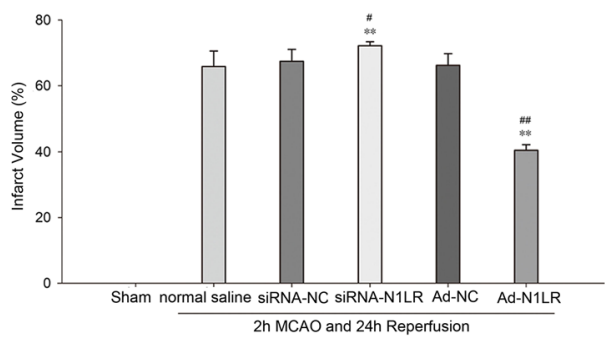

$\mathrm{h}_{\text {sham }}$
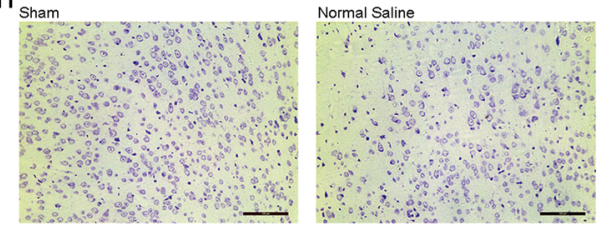

SiRNA-NC

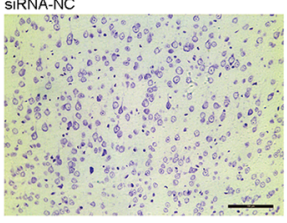

SiRNA-N1LR

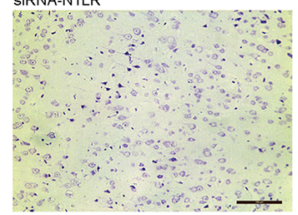

Ad-NC

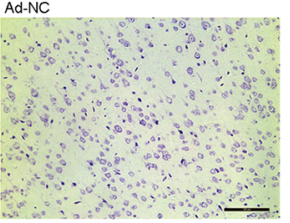

Ad-N1LR
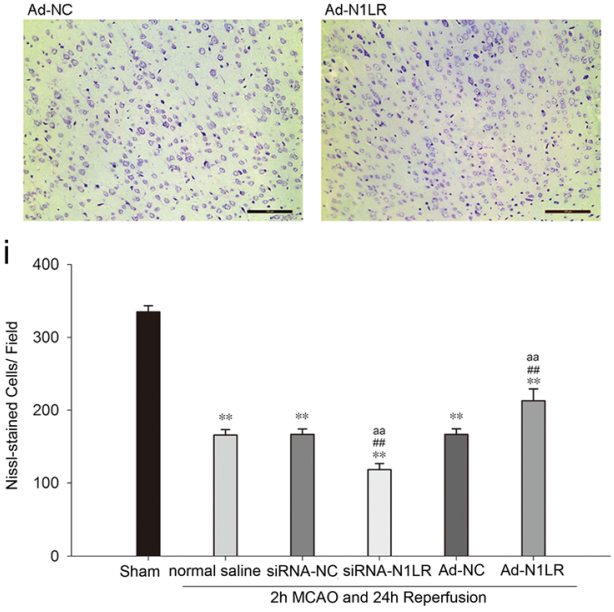